\title{
Universal High-Energy Photoelectron Emission from Nanoclusters Beyond the Atomic Limit
}

\author{
Zhou Wang, ${ }^{1}$ Abraham Camacho Garibay $\odot,{ }^{1,2}$ Hyunwook Park, ${ }^{1}$ Ulf Saalmann $\odot,{ }^{2}$ \\ Pierre Agostini, ${ }^{1}$ Jan M. Rost ${ }^{2}$ and Louis F. DiMauro, ${ }^{1, *}$ \\ ${ }^{1}$ Department of Physics, The Ohio State University, Columbus, Ohio, USA \\ ${ }^{2}$ Max Planck Institute for the Physics of Complex Systems, Nöthnitzer Straße 38, 01187 Dresden, Germany
}

(Received 14 August 2019; revised manuscript received 4 March 2020; accepted 3 April 2020; published 29 April 2020)

\begin{abstract}
Rescattering by electrons on classical trajectories is central to understand photoelectron and highharmonic emission from isolated atoms or molecules in intense laser pulses. By controlling the cluster size and the quiver amplitude of electrons, we demonstrate how rescattering influences the energy distribution of photoelectrons emitted from noble gas nanoclusters. Our experiments reveal a universal dependence of photoelectron energy distributions on the cluster size when scaled by the field driven electron excursion, establishing a unified rescattering picture for extended systems with the known atomic dynamics as the limit of zero extension. The result is supported by molecular dynamics calculations and rationalized with a one-dimensional classical model.
\end{abstract}

DOI: 10.1103/PhysRevLett.124.173201

Photoelectron spectroscopy is one of the most versatile and powerful methods to investigate matter. In the wellknown photoeffect, weak radiation leads to prompt ionization of an electron with binding energy $E_{b}$ through absorption of a single photon. This direct emission of photo electrons is also the dominating process if the irradiating laser field is very strong, such that $U_{p} \gtrsim E_{b}$, where $U_{p}=F^{2} /\left(4 \omega^{2}\right)$, which is the average energy of a free electron in the laser field of peak amplitude $F$ and frequency $\omega$. During a laser cycle, the electromagnetic force can be so strong that it drives the electron back to the ion where it rescatters. Such events are rare but can lead to considerable additional energy for the photoelectron of up to $10 U_{p}$. For the electron to reach this characteristic maximal energy requires release and rescattering at specific phases of the oscillating laser field [1-3]. On the other hand, in this so-called above-threshold-ionization (ATI) process the maximal energy is independent of details in the atomic or molecular target electron distributions. This can be understood from the observation that the distributions with an extension of a few atomic units are essentially pointlike compared to the distance of $x_{\omega}=F / \omega^{2}$ quantifying the excursion of a free electron in the strong laser field ruling the rescattering process. Remarkably, strong field electron dynamics can be described to a large extent by

Published by the American Physical Society under the terms of the Creative Commons Attribution 4.0 International license. Further distribution of this work must maintain attribution to the author(s) and the published article's title, journal citation, and DOI. Open access publication funded by the Max Planck Society. classical electron trajectories which facilitates the understanding of the process, as well as interpretation and modeling of experimental results.

In the following, we will investigate if and how this rescattering induced photoionization applies to extended targets such as nanoclusters or nanoparticles [4-6] with sizes of the same order of magnitude as $x_{\omega}$. While previously the production of energetic electrons in extended systems was understood as a thermalization process [7] not linked to strong field rescattering, recent experiments on argon clusters [8] and field-controlled photoemission from dielectric nanospheres [4], as well as theoretical considerations [9] have revealed a characteristic feature of rescattering: the energetic photoelectrons exhibit a strong dependence on the phase of the electromagnetic field. Encouraged by this observation, we will present here a unified rescattering picture valid from atoms to extended systems for high-energy electrons generated in the interaction with an intense laser pulse.

In our work, an intense laser, tunable from near-infrared (NIR, $0.8 \mu \mathrm{m}$ ) to midinfrared (MIR, $1.8 \mu \mathrm{m}$ ) wavelengths, is used to generate a well-defined field driven electronic excursion distance $x_{\omega}$ relative to the target's radius $R_{\mathrm{cl}}$, which is varied by creating clusters from a single up to $10^{5}$ argon atoms. The photoelectron energy distribution (PED) is measured with a time-of-flight spectrometer along the laser polarization. PEDs from nanoclusters are compared with back-to-back measurements from argon monomers under identical laser conditions. The nanocluster size is controlled by the stagnation pressure of the cluster valve and estimated according to the Hagena empirical formula [10-12]. 

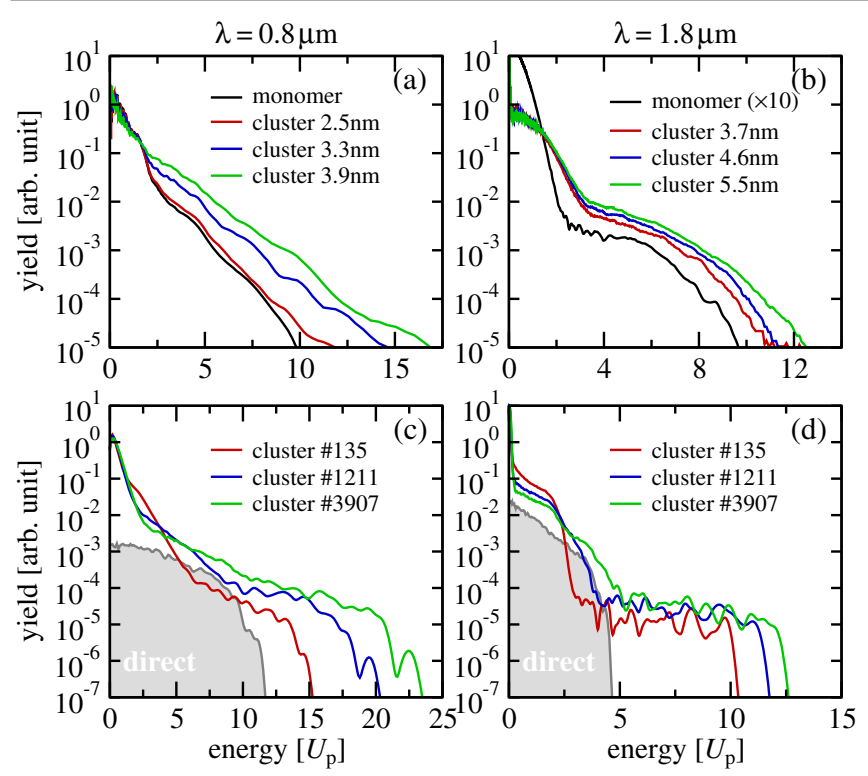

FIG. 1. Photoelectron energy distributions from experiments (a),(b) and MD calculations (c),(d) for the argon atom (black) and different sizes of argon clusters specified in the figures. For clarity, all spectra are normalized to area one. Experimental data are taken at $I=170 \mathrm{TW} / \mathrm{cm}^{2}$ for both $0.8\left(U_{p} \sim 10 \mathrm{eV}\right)$ (a) and $1.8 \mu \mathrm{m}\left(U_{p} \sim 51 \mathrm{eV}\right)(\mathrm{b})$, respectively. MD calculations are performed at $I=600 \mathrm{TW} / \mathrm{cm}^{2}$ for both $0.8\left(U_{p} \sim 36 \mathrm{eV}\right)$ (c) and $1.8 \mu \mathrm{m}\left(U_{p} \sim 182 \mathrm{eV}\right)(\mathrm{d})$, respectively. The PED spectra shaded in gray and marked "direct" are from calculations with 3907 atoms without hard recollisions of the photoelectrons. The experimental cluster size range is estimated to be $\Delta R \approx\langle R\rangle / 3$.

Figures 1(a) and 1(b) provide the measured PEDs for monomers and clusters of different size. The measured PED for monomers (black line) reaches up to $10 U_{p}$, indicative of the maximum energy from rescattering, where an ionized electron is accelerated by the laser field and driven back to collide with the parent ion $[18,19]$. In contrast, the PEDs from nanoclusters differ from the monomer counterpart, with photoelectron energies increasingly beyond $10 U_{p}$ with growing nanocluster size. At a longer wavelength of $1.8 \mu \mathrm{m}$ [Fig. 1(b)] the standard rescattering features become more pronounced in the monomer PED showing a break at $2 U_{p}$ and a cutoff at $10 U_{p}$ [3]. The photoelectrons below $2 U_{p}$ result from direct (tunneling) ionization without rescattering, while those in the plateau up to the $10 U_{p}$ limit are from field-driven rescattering. In contrast to the $0.8 \mu \mathrm{m}$ case, the nanocluster PEDs at $1.8 \mu \mathrm{m}$ show similar features as the atomic one. The strikingly analogous plateau structure suggests a mechanism rooted in rescattering also for clusters. Similarly as observed for $0.8 \mu \mathrm{m}$, the PE spectra at $1.8 \mu \mathrm{m}$ progressively extends beyond $10 U_{p}$ with the cluster size albeit with a smaller increment, despite the fact that the maximal nanocluster in Fig. 1(b) is larger than in Fig. 1(a).
Early results on the interaction of clusters with infrared laser light were interpreted as stochastic energy absorption through an inverse-Bremsstrahlung process [7]. In this scenario the electrons randomly collide with the ions inside the nanoclusters (and among themselves) and thermalize. Consequently, their energies follow a Maxwell-Boltzmann distribution, which was characterized by a temperature $[13,20,21]$. Such stochastic energy absorption has no relation to the phase of the laser field, whereas rescattering-dominated dynamics depends sensitively on it. Therefore, we have performed two-color $(\omega, 2 \omega)$ measurements in order to reveal the phase dependence of the energy absorption in the clusters.

To this end the fundamental driving field $(\omega)$ at either NIR $(0.8 \mu \mathrm{m})$ or MIR $(1.7 \mu \mathrm{m})$ wavelength is frequency doubled to generate a weak $(\sim 3 \%$ of $\omega)$, phase-locked second harmonic $(2 \omega)$ field. The beams with temporal and spatial overlap are focused to interact with the targets. The instantaneous total electric field of the synthesized beam can be controlled by the phase delay $\phi$ between the two fields. Figures 2(a) and 2(b) show the delay-dependent PEDs from argon atoms. As can be seen the atomic cutoff oscillates with $\phi$ characteristic for rescattering in atoms $[22,23]$ for both fundamental wavelengths. For clusters under identical conditions, as shown in Figs. 2(c) and 2(d) similar oscillations in the high-energy photoelectron yield can be clearly identified with a nearly identical phase dependence. Note that in the two-color measurements, the input energy, average intensity, and pulse duration are kept constant with respect to $\phi$. Hence, inverse Bremsstrahlung
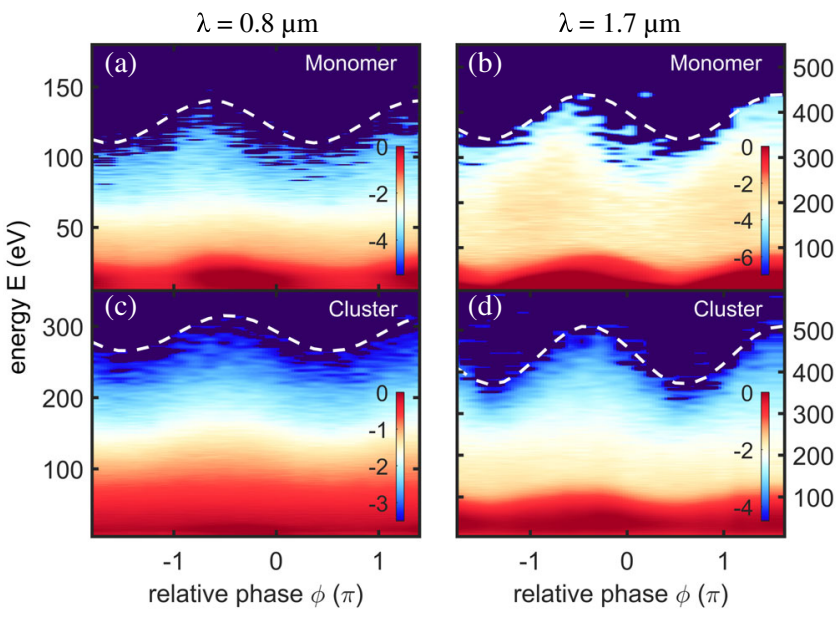

FIG. 2. Two-color $(\omega, 2 \omega)$ measurement of the photoelectron energy from argon atoms and argon clusters $\left(R_{\mathrm{cl}} \sim 3.2 \mathrm{~nm}\right)$ at base wavelength 0.8 (corresponding to $\omega_{1}=0.057$ a.u., left panels) and $1.7 \mu \mathrm{m}\left(\omega_{2}=0.027\right.$ a.u., right panels $)$. Intensities for each color are $I_{\omega_{i}} \sim 140$ and $I_{2 \omega_{i}} \sim 5 \mathrm{TW} / \mathrm{cm}^{2}$ for $i=1,2$. The PEDs are resolved with respect to the delay $\phi$ between fundamental $\mathcal{E}_{\omega}$ and the copolarized second harmonic field $\mathcal{E}_{2 \omega}$ with $\mathcal{E}_{\text {total }}=\mathcal{E}_{\omega_{i}} \cos \left(\omega_{i} t\right)+\mathcal{E}_{2 \omega_{i}} \cos \left(2 \omega_{i} t+\phi\right)$. White dashed lines are presented as a help to guide the eye. 
can be ruled out as an origin of the high-energy cluster photoelectrons. Rather, the almost perfect synchronization between the cutoff modulations in atoms and the corresponding high-energy modulations in clusters point to rescattering as a source for this phenomenon, regardless of the wavelength. This conclusion is consistent with cluster PED measurements using few-cycle MIR pulses [8] and carrier-envelope phase measurements on dielectric nanospheres [4]. We note that many-electron effects in clusters considerably suppress $\phi$ modulation for lowenergy electrons [Figs. 2(c) and 2(d)]. These are attributed to thermalized electrons whose emission occurs at later times when the pulse is no longer present, in contrast to those from atoms [Figs. 2(a) and 2(b)] where such a mechanism is not available.

These PEDs from different cluster sizes at wavelengths 0.8 and $1.8 \mu \mathrm{m}$ are plotted in Figs. 1(c) and 1(d), respectively. The simulated PEDs, for single-size clusters without focal averaging, exhibit the observed experimental features and trends qualitatively. For both wavelengths, the spectra progressively increase beyond $10 U_{p}$ with growing cluster size, with a larger effect for $0.8 \mu \mathrm{m}$ than for $1.8 \mu \mathrm{m}$, as in the experimental spectra of Figs. 1(a) and 1(b). If hard recollisions are excluded from the simulation as it was done for the black curves enclosing gray-shaded areas in Figs. 1(c) and 1(d), the distributions resemble PEDs familiar from atoms for "direct" electrons, although such electrons can acquire energies beyond $2 U_{p}$, the classical limit for direct electrons in atoms [9], due to the cluster environment. Hence, the simulated PEDs and, in particular, the comparison with the direct processes, also point to recollisions as the source for the high-energy electrons.

In contrast to atoms, rescattering in extended objects such as clusters should depend on the two physical spatial scales involved, the nanocluster size described by the cluster radius $R_{\mathrm{cl}}$, and the electron quiver distance $x_{\omega}$. In a typical strong-field experiment, $x_{\omega}$ is on the order of a few nanometers, which is significantly larger than the extension of the ground-state electron density in an atom or molecule. Consequently, the standard rescattering model assumes that the collision occurs at the atomic origin and-in its simplest version - that the external field rules the electron motion rendering the Coulomb field negligible, especially at high energy. If small, relative to the extension of the quiver distance $\left(R_{\mathrm{cl}} \ll x_{\omega}\right)$, the cluster appears in the strong field dynamics as a point scatterer, thus manifesting atomiclike scattering. On the other hand, if the cluster size is comparable to $x_{\omega}$, as in our experiment in particular for $0.8 \mu \mathrm{m}$ light $\left(x_{\omega}=1.12 \mathrm{~nm}\right.$ in this case), differences in the recollision dynamics can be expected as electrons may rescatter from any ion or atom in the cluster. This suggests that the electronic energy distribution can be formulated as a function of the dimensionless cluster radius $R=R_{\mathrm{cl}} / x_{\omega}$, which takes into account our intuition that high-energy photoelectrons from a cluster behave like those of an atom under a strong laser field if the quiver distance of the electron is much larger than the cluster radius.

To establish the high-energy behavior of electrons in clusters it would be desirable to track characteristic electron trajectories in order to reveal their energy gaining process as in the atomic case. At first glance, however, it is impossible to identify a simple mechanism ruling the maximal energy gain in a nanocluster due to its complexity. Yet, it is clear that an energetic backscattered electron is so fast that it will promptly leave the cluster without any further interaction. This means that the vast majority of the electron energy is obtained through a single "last" violent collision while every other previous particle-particle interaction can essentially be neglected. The corresponding trajectory after the "last return" (i. e., an electron being driven back to the cluster by the laser field) can be calculated classically, and seamlessly connects to the three-step model from atoms and molecules $[18,19]$.

To be able to relate cluster rescattering to the original atomic scenario, we have devised a $1 \mathrm{D}$ rescattering model which contains the atomic case as a limit (see Supplemental Material [12]). The nanocluster is represented by a square potential with a width of $2 R_{\mathrm{cl}}$ and a depth of $V$. Furthermore, we assume that the cluster gets ionized until the laser field with peak amplitude $F$ is no longer able to remove electrons of the $Q$-fold charged cluster which retains electrons at the surface with its Coulombic force. This happens if $Q / R_{\mathrm{cl}}^{2}=F[9,24]$. Thus we can replace $Q$ in the potential depth $V=Q / R_{\mathrm{cl}}$ with $F R_{\mathrm{cl}}^{2}$, which allows us to define a scaled Hamiltonian

$$
h(R)=H\left(R_{\mathrm{cl}} / x_{\omega}\right) / U_{p} .
$$

The dynamics driven by $h(R)$ does not depend on the laser parameters (intensity and frequency) explicitly. To determine the photoelectron cutoff in the model, an electron is released from the edge $R_{\mathrm{cl}}$ of the cluster at any initial time (or phase $\phi_{0}$ of the laser field, respectively), accelerated by the field, and driven back to scatter at any location $x_{r}$ within the cluster $\left|x_{r}\right|<R_{\mathrm{cl}}$. The final electron energy $E\left(\phi_{0}, x_{r} ; R_{\mathrm{cl}}, x_{\omega}\right)$ is maximized numerically with respect to $\phi_{0}$ and $x_{r}$ which gives the dependence of the scaled cutoff energy on the scaled cluster radius $R$ shown as a gray line in Fig. 3. As one can see, it follows the results from the MD calculations (open symbols) remarkably well, indicating that the dependence on further properties (such as pulse length or energy gain through residual collisions) is weak for the parameters chosen. The open symbols show the photoelectron events with the largest energy from the MD calculations (for details see the Supplemental Material [12]) corresponding to the evolution of the atomic cutoff as a function of cluster size. Thereby the MD results represent calculations for different cluster sizes, photon energies, and pulse intensities underscoring the validity of the scaling. 


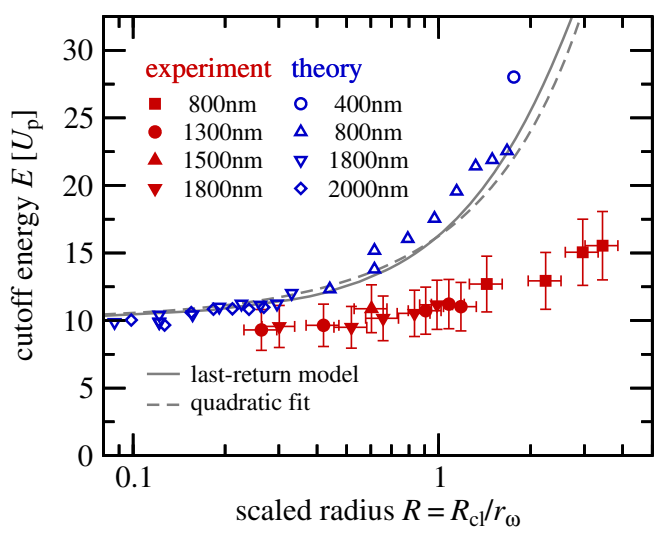

FIG. 3. Evolution of different cutoffs with the scaled cluster radius $R$. Experimental values (solid symbols) are obtained though the condition $P\left(E^{\text {cutoff }}\right)=k P\left(5 U_{\mathrm{p}}\right)$, with $k=10^{-3} \ldots 10^{-2}$, depending on the wavelength. MD simulation values (open symbols) give the highest energies recorded. The gray-solid line shows the results from the 1D "last return" model (see text), the gray-dashed line is a quadratic fit according to Eq. (2) to it. Data are taken in the range of $I=150 \ldots .500 \mathrm{TW} / \mathrm{cm}^{2}, R_{\mathrm{cl}}=0.9 \ldots 7.8 \mathrm{~nm}$, and $T=45 \ldots 60 \mathrm{fs}$ in the experiment and $I=600 \mathrm{TW} / \mathrm{cm}^{2}$, $R_{\mathrm{cl}}=1.0 \ldots 3.2 \mathrm{~nm}$, and $T=60 \mathrm{fs}$ in the MD calculations.

In the small cluster or long wavelength limit, the electron traverses the entire cluster within a small fraction of an optical cycle and therefore without any significant change in the field phase, and hence the cutoff energy remains around $10 U_{p}$ as in the atomic case for $R=R_{\mathrm{cl}} / x_{\omega} \ll 1$. The depth of the potential plays a minor role for the parameters considered here since the energy for the cutoff is dominated by the drift momentum $F / \omega$. In the large cluster or short wavelength limit, we leave the "atomic regime," and the electron will rescatter from an atom inside the cluster for which the field phase is such that the energy gained from this process is maximized. For increasing $R$ the potential depth $V / U_{p}$ becomes more important than the drift momentum, since it increases the velocity with which the electron suffers elastic rescattering in the cluster and therefore amplifies the energy gain. Over the energy range covered in Fig. 3 the simple form

$$
E^{\max }(R) \approx 10 U_{p}(1+a R)^{2},
$$

with $a=0.275$ (shown as a dashed line in Fig. 3) parametrizes the size-dependent high-energy scale in terms of the theoretical cutoff for our argon clusters very well. We should point out that this fit is only valid over the size range presented here, and that the parameter $a$ has no deeper meaning.

For atoms the $10 U_{p}$ cutoff sets the energy scale for the high-energy part of the PEDs. We see from Eq. (2) that for clusters the scale is amended by the factor $(1+a R)^{2}$, depending in a simple way on the scaled cluster radius $R=R_{\mathrm{cl}} / x_{\omega}$. If the high-energy behavior of the rescattered photoelectrons is universal, with the help of the scaled radius $R$ computed from experimental conditions the
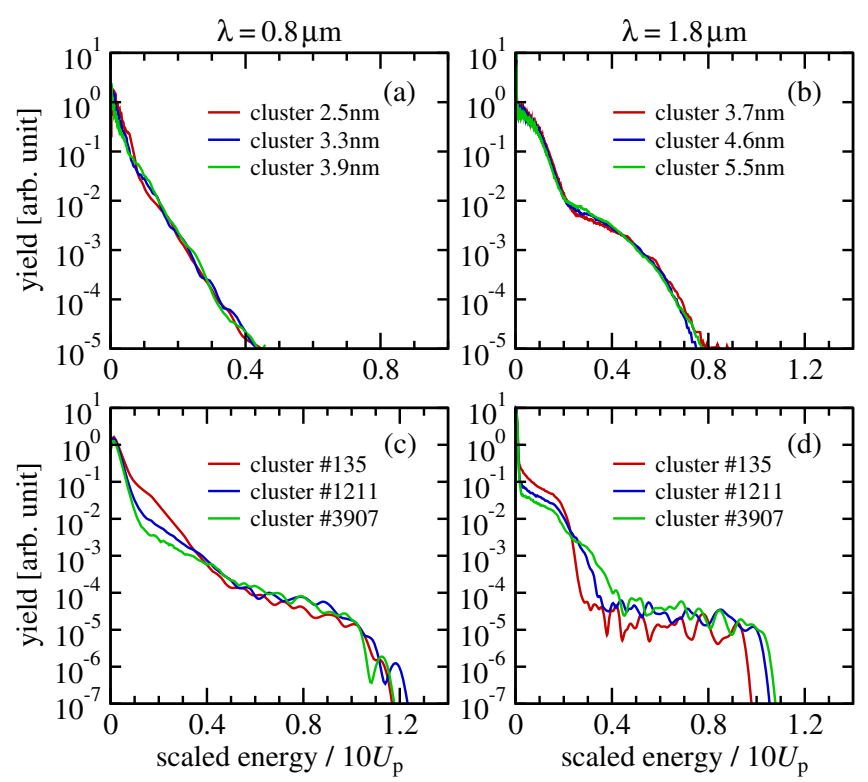

FIG. 4. Photoelectron energy distributions for argon clusters as in Fig. 1 from experiments (a),(b) and MD calculations (c),(d), but with the energy axis rescaled according to the energy scale $E^{\max }(R)$ from Eq. (2).

spectra shown in Fig. 1 should collapse to a single curve when plotted against the scaled photoelectron energy $E / E^{\max }(R)$. In Fig. 4 one can see that this is indeed the case for both the experimental and the theoretical spectra.

In general the maximal energies reached in the experimental spectra are smaller than in the theoretical ones. This is, however, to be expected as the dynamic range of the yield in the experiment is significantly smaller than in the calculations. Yet, reading off values from the experimental spectra close to the noise detection limit by using the condition $P\left(E^{\text {cutoff }}\right)=k P\left(5 U_{p}\right)$, with $k$ ranging from $10^{-3}$ to $10^{-2}$ depending on the wavelength, from the experimental spectra close to the noise detection limit reveals once again the universal high-energy rescattering behavior, see full (red) symbols in Fig. 3. Consistent with the experimentally determined cutoff the rise of the experimental curve with $R$ is weaker than for the theoretical cutoff [black line and open (blue) symbols]. Comparison with the theoretical result suggests that a noise threshold at least reduced by a factor 100 would be necessary to reveal the cluster cutoff. However, the cutoff is only one, albeit wellknown, consequence of strong-field rescattering dynamics. As we have demonstrated in this work with the help of size varying clusters, strong field rescattering dynamics is characterized by a universal high-energy behavior of photoelectron distributions over wide range of energies revealed by the $R$-dependent energy scale function (2). This remarkable property has so far gone unnoticed, since for pointlike systems relative to the quiver motion, such as atoms and molecules, the scale function reduces to the well-known single point, $E^{\max }(0)=10 U_{p}$. 
Finally, to understand qualitatively the limits of this universal behavior, we recall the conditions under which it was demonstrated. The mean-free-path length for the energetic electrons should be large enough to ensure that the photo-electron does not undergo further collisions after the violent one which lead to its decisive energy gain. Furthermore, in contrast, e.g., to the scenario of Ref. [4] the pulse must be long enough such that saturation in the charging is reached in order to have $Q / R_{\mathrm{cl}}^{2}=F$, which allows us to formulate the scaled Hamiltonian (1). Last but not least, the scale function (2) will have a more complicated form if the Coulomb potential of the cluster is relevant, which applies to large charging $Q$. This is, however, beyond the scope of this Letter.

In conclusion, by experiments controlling cluster size and laser parameters of intense pulses interacting with argon clusters, we have revealed that a universal highenergy photoelectron regime exists, which is characterized by hard recollisions of laser driven electrons from ions or atoms in the cluster in analogy to atoms. Universality emerges as the high-energy scale depends only on a combination of cluster size and laser parameters, namely, the ponderomotive energy and the cluster radius scaled by the quiver amplitude $R=R_{\mathrm{cl}} / x_{\omega}$, verified within the limits of experimental constraints and uncertainties in this experiment. We expect this characteristic length scale to be also relevant for intense light-driven processes in nanostructures or large polyatomic biomolecules.

This material is based upon work supported by the Air Force Office of Scientific Research under MURI Grant No. FA9550-16-1-0013 and by the National Science Foundation under Grant No. PHY-1605042. The DiMauro group acknowledges support from the Ohio Supercomputer Center through Project No. PAS0207.

Z.W. and A. C. G. contributed equally to this work.

*dimauro.6@osu.edu

[1] G. G. Paulus, W. Becker, W. Nicklich, and H. Walther, Rescattering effects in above-threshold ionization: A classical model, J. Phys. B 27, L703 (1994).

[2] J. Tate, T. Auguste, H. G. Muller, P. Salieres, P. Agostini, and L. F. DiMauro, Scaling of Wave-Packet Dynamics in an Intense Midinfrared Field, Phys. Rev. Lett. 98, 013901 (2007).

[3] P. Colosimo et al., Scaling strong-field interactions towards the classical limit, Nat. Phys. 4, 386 (2008).

[4] S. Zherebtsov et al., Controlled near-field enhanced electron acceleration from dielectric nanospheres with intense fewcycle laser fields, Nat. Phys. 7, 656 (2011).

[5] F. Süßmann et al., Field propagation-induced directionality of carrier-envelope phase-controlled photoemission from nanospheres, Nat. Commun. 6, 7944 (2015).
[6] M. F. Ciappina et al., Attosecond physics at the nanoscale, Rep. Prog. Phys. 80, 054401 (2017).

[7] T. Ditmire, T. Donnelly, A. M. Rubenchik, R. W. Falcone, and M.D. Perry, Interaction of intense laser pulses with atomic clusters, Phys. Rev. A 53, 3379 (1996).

[8] B. Schütte, P. Ye, S. Patchkovskii, D. R. Austin, C. Brahms, C. Strüber, T. Witting, M. Y. Ivanov, J. W. G. Tisch, and J. P. Marangos, Strong-field ionization of clusters using twocycle pulses at $1.8 \mu \mathrm{m}$, Sci. Rep. 6, 39664 (2016).

[9] U. Saalmann and J. M. Rost, Rescattering for Extended Atomic Systems, Phys. Rev. Lett. 100, 133006 (2008).

[10] O. F. Hagena, Cluster ion sources (invited), Rev. Sci. Instrum. 63, 2374 (1992).

[11] F. Dorchies, F. Blasco, T. Caillaud, J. Stevefelt, C. Stenz, A. S. Boldarev, and V. A. Gasilov, Spatial distribution of cluster size and density in supersonic jets as targets for intense laser pulses, Phys. Rev. A 68, 023201 (2003).

[12] See Supplemental Material at http://link.aps.org/ supplemental/10.1103/PhysRevLett.124.173201 for further details and discussion, which contains Refs. [10,13-17].

[13] E. Springate, S. A. Aseyev, S. Zamith, and M. J. J. Vrakking, Electron kinetic energy measurements from laser irradiation of clusters, Phys. Rev. A 68, 053201 (2003).

[14] Y. L. Shao, T. Ditmire, J. W. G. Tisch, E. Springate, J. P. Marangos, and M.H. R. Hutchinson, Multi-keV Electron Generation in the Interaction of Intense Laser Pulses with Xe Clusters, Phys. Rev. Lett. 77, 3343 (1996).

[15] U. Even, The Even-Lavie valve as a source for high intensity supersonic beam, EPJ Tech. Instrum. 2, 17 (2015).

[16] D. W. Schumacher and P. H. Bucksbaum, Phase dependence of intense-field ionization, Phys. Rev. A 54, 4271 (1996).

[17] S. Alvarez, A cartography of the van der Waals territories, Dalton Trans. 42, 8617 (2013).

[18] K. J. Schafer, B. Yang, L. F. DiMauro, and K. C. Kulander, Above Threshold Ionization Beyond the High Harmonic Cutoff, Phys. Rev. Lett. 70, 1599 (1993).

[19] P. B. Corkum, Plasma Perspective on Strong Field Multiphoton Ionization, Phys. Rev. Lett. 71, 1994 (1993).

[20] V. Kumarappan, M. Krishnamurthy, and D. Mathur, Twodimensional effects in the hydrodynamic expansion of xenon clusters under intense laser irradiation, Phys. Rev. A 66, 033203 (2002).

[21] V. Kumarappan, M. Krishnamurthy, and D. Mathur, Asymmetric emission of high-energy electrons in the two-dimensional hydrodynamic expansion of large xenon clusters irradiated by intense laser fields, Phys. Rev. A 67, 043204 (2003).

[22] D. Ray, Z. Chen, S. De, W. Cao, I. V. Litvinyuk, A. T. Le, C. D. Lin, M. F. Kling, and C. L. Cocke, Momentum spectra of electrons rescattered from rare-gas targets following their extraction by one- and two-color femtosecond laser pulses, Phys. Rev. A 83, 013410 (2011).

[23] S. Skruszewicz, J. Tiggesbäumker, K.-H. Meiwes-Broer, M. Arbeiter, T. Fennel, and D. Bauer, Two-Color Strong-Field Photoelectron Spectroscopy and the Phase of the Phase, Phys. Rev. Lett. 115, 043001 (2015).

[24] U. Saalmann, Resonant energy absorption of rare-gas clusters in strong laser pulses, J. Mod. Opt. 53, 173 (2006). 